\title{
Medios audiovisuales de servicio público y desinformación. Principales políticas y estrategias contra las noticias falsas
}

\section{Public service audiovisual media and disinformation. Main policies and strategies against fake news}

Ana María López Cepeda - Universidad de Castilla-La Mancha : ana.lopezcepeda@uclm.es

María José Ufarte Ruiz - Universidad de Castilla-La Mancha mariajose.ufarte@uclm.es

Francisco José Murcia Verdú - Universidad de Castilla-La Mancha francisco.murcia@uclm.es

Abstract: El incremento de noticias falsas, que tienen como objetivo distorsionar la realidad y generar confusión entre la verdad y la mentira, se ha convertido en una problemática social a nivel global. La verificación de la información ha surgido como elemento fundamental para la profesión periodística y ya se han lanzado algunas experiencias desde diversos medios de comunicación, plataformas y organizaciones. La Unión Europea ha presentado un Plan de Acción - en vigor desde 2019- para contrarrestar cualquier campaña de posverdad, basado en un incremento de los recursos, la coordinación, la elaboración de un código de buenas prácticas y la creación de grupos de verificación y contraste de datos. En este contexto, el papel de los medios audiovisuales de titularidad pública no tiene que ser secundario y debe liderar un proceso de alfabetización mediática coordinado en cada Estado miembro para recortar el incremento de la desinformación. El objetivo de esta investigación es analizar las políticas y estrategias que las principales 


\section{Las nuevas narrativas, en el entorno social \\ Universidad de La Laguna, diciembre de 2019}

radiotelevisiones públicas europeas están aplicando para potenciar la lucha contra las noticias falsas. El diseño metodológico para alcanzar este propósito parte de la revisión sistemática de la literatura científica, que ha permitido conocer las principales aportaciones al estado de la cuestión y generar un relato sobre este tema, que es de interés creciente en el área de Comunicación pero aún no exhaustivamente abordado. Además, se analiza la literatura gris (memorias, informes y otros documentos) y acciones desarrolladas por las televisiones públicas objeto de estudio. Los resultados revelan que se perfilan nuevas políticas y estrategias en los medios audiovisuales de servicio público asociadas a la lucha contra la desinformación y las noticias falsas.

Keywords: Medios audiovisuales; servicio público; noticias falsas; estrategias; desinformación; verificación.

\section{Introducción}

El nuevo ecosistema mediático es un campo propicio para la información errónea, imprecisa, malintencionada, inventada y no fundamentada, que induce a error en los lectores y entorpece la actividad de quien quiere informarse correctamente (Zommer, 2014). Son las noticias falsas o fake news, según la terminología inglesa, que alude a aquellas informaciones distorsionadas (Khaldarova; Pantti, 2016), con altos niveles de facticidad y engaño (Tandoc; Lim, Ling, 2018), que se modifican, ridiculizan y se someten a nuevas interpretaciones (Balmas, 2012) para favorecer intereses ideológicos o económicos (Allcott; Gentzkow, 2017).

Para Rubin, Chen y Conroy (2016), este concepto también engloba las noticias elaboradas por la prensa sensacionalista que cuentan con titulares no contrastados y exageraciones, los bulos a gran escala que se han construido de forma deliberada para engañar a la audiencia, y las noticias falsas humorísticas que se elaboran siguiendo los preceptos de las noticias tradicionales. Por su parte, Tandoc, Lim y Ling (2018) consideran que igualmente hay que tener en cuenta las noticias satíricas, las parodias, los contenidos falsos redactados de manera periodística que no son advertidos a 
la audiencia, la manipulación de imágenes, los contenidos publicitarios bajo la apariencia de informes y la propaganda.

Derakhshan y Wardle (2017) destacan la intencionalidad como un factor crucial para entender este fenómeno, mientras que Zaryan (2017) sostiene que lo realmente importante es que las personas accedan a esa información y la compartan, en un momento en el que las formas y posibilidades de informarse han cambiado, especialmente entre los jóvenes (López-Vidales; González Aldea; Medina de la Viña, 2011). Sin embargo, para Catalina-García, Sousa y Silva (2019) el problema radica en si las audiencias son capaces de diferenciar las noticias falsas de las que no lo son, ya que con frecuencia dan credibilidad a las informaciones que mejor encajan con su visión del mundo (Mihailidis; Viotty, 2017).

Las consecuencias, en cualquier caso, son negativas porque se confunde la verdad con la mentira, se incrementa la credibilidad hacia lo falso (Rapp; Salovich, 2018) y se pone en riesgo el discurso democrático (Champy; 2011; Codeluppi, 2018; Fisher, 2018; McChesney, 2014). Pero este fenómeno no constituye una característica propia y exclusiva del momento actual (Petroni; Massa; Anzera, 2017). Se trata de una vieja práctica que ha estado muy presente a lo largo de la historia de la Comunicación, especialmente durante los grandes conflictos bélicos y en las etapas de entreguerras para favorecer determinados intereses (Schudson; Zelizer, 2017). Sin embargo, ha adquirido un nuevo cariz en el momento actual, sobre todo con la eclosión de las redes sociales (Haigh; Haigh; Kozak, 2017; Watts; Rothschild, 2017) y después de que Donald Trump pusiera en duda la veracidad de las informaciones de algunos medios de comunicación durante su campaña electoral en 2016 (Jankowski, 2018; Greenberg, 2017).

Como respuesta a esta crisis de confianza, se han puesto en marcha diferentes proyectos, políticas y estrategias destinados a comprobar a posteriori las informaciones publicadas por los medios de comunicación, así como las declaraciones o comentarios realizados por líderes políticos o personajes relevantes. Para Amorós (2018) y Mantzarlis (2018) es la mejor forma para contrarrestar el poder de las noticias falsas y ha sido ideada por el periodismo 


\section{Las nuevas narrativas, en el entorno social \\ Universidad de La Laguna, diciembre de 2019}

para combatirlas y ofrecer a la ciudadanía un relato completamente ajustado a la realidad.

El origen de estas estrategias centradas en la verificación de la información como práctica institucionalizada se remonta a 1913, cuando el diario New York World fundó el Bureau of Accuracy and Fair Play, un organismo dedicado a corregir descuidos y erradicar a los farsantes (Ayuso; Bauzá, 2018). El objetivo, en cualquier caso, es contribuir de forma más eficaz a la exigencia de responsabilidad de los representantes públicos y a la mejor información de los ciudadanos, un punto clave para fortalecer la democracia (Gueham, 2017).

En la actualidad, estas prácticas se han popularizado y estas iniciativas se han multiplicado a nivel nacional e internacional (Palau Sampio, 2018; Spivak, 2011; Stencel, 2016), a pesar de que la labor de confirmar y comprobar hechos existe desde el inicio del periodismo como un elemento para garantizar la calidad.

\section{Mapa de medios con políticas y estrategias contra las noticias falsas}

En los últimos años, muchos medios de comunicación y plataformas independientes han puesto en marcha secciones, grupos de trabajo o espacios dirigidos a la verificación de informaciones (Cherubini; Graves, 2016). El censo de proyectos elaborado por el Reporters' Lab de la Duke University (Duke Reporter's Lab, 2019) cuantifica hasta el 1 de noviembre de 2019 un total de 269 iniciativas en 62 países, de las que 195 están activas y 74 inactivas. Son 143 iniciativas más que en junio de 2017, cuando la institución contabilizó 126 webs y organizaciones dedicadas a estas funciones, un $24 \%$ más que el año anterior y el doble que las contabilizadas en 2015 (Adair; Thakore, 2015).

Vázquez Herrero, Vizoso y López García (2019) también han realizado una exploración mundial de proyectos e iniciativas dedicas a combatir las noticias falsas y señalan que, atendiendo al origen geográfico, Europa -con 51 iniciativas- es el continente con mayor representación, aunque también se identifican casos en Norteamérica, Asia, Sudamérica, África y Oceanía. Los autores apuntan que el país con mayor número de políticas y estrategias es Estados Unidos -31 en total-, junto a Francia, India, Brasil, Indonesia y Reino 
Unido, todos ellos con al menos 5 entidades de verificación. Sin embargo, en opinión de Graves (2016) el panorama de proyectos asociados a la verificación de información desafía cualquier intento de categorización.

En EE.UU., por ejemplo, destaca FactCheck.org (www.factcheck.org), una iniciativa de la Universidad de Pensilvania que se anuncia como un defensor de los votantes no partidista y sin ánimo de lucro. También está la web Politifact.com (www.politifact.com), una de las más famosas del país que nació de la mano de Bill Adair, reportero de Tampa Bay Times en Washington y ganador de un premio Pulitzer; y el portal politicalcorrection.com (www.politicalcorrection.com). Los periódicos más prestigiosos del país también se han apuntado a este fenómeno. The Washington Post incluye, desde 2007, tanto en su edición digital como en la de papel, un espacio informativo denominado The Fact Checker, en el que se evalúan los hechos y declaraciones de los candidatos.

En América Latina, concretamente en Argentina, destaca Chequeado (www.chequeando.com), que apuesta por la verificación periodística de los hechos y declaraciones; y El Mercurio de Chile revisa los datos gracias a EI Polígrafo. En Costa Rica, el semanario El Financiero dispone de la aplicación Rete al candidato y Honduras, de El Observador. En México, el portal verificado.mx ha sido de gran ayuda para la ciudadanía al revisar y analizar datos e informaciones que circulan en las redes sociales en los periodos electorales más importantes del país. En África está la plataforma Áfricacheck (www.africacheck.org), que ofrece consejos, guías, documentos, e incluso premios de verificación a aquellos periodistas que mejor desempeñaron su labor en este campo.

En el ámbito europeo, Ucrania dispone del portal Fake Control (www.fakecontrol.org), que analiza «desde noticias de política internacional a los rumores de la ciudad", como indican en su web. Reino Unido apuesta por la verificación de datos a través del blog The fact Checking Blog, de Channel 4, y Fullfac (https://fullfact.org/). Y en Francia, los periódicos Libération y Le Monde abrieron el paso a estas iniciativas con las secciones Désintox (2008) y Les Décodeurs (2009), mientras que Alemania tiene su propio watchblog 
gracias a BILDblog (http://www.bildblog.de/). En Italia destaca la web Pagella Politica.

Siguiendo la tendencia internacional, algunos medios españoles se han enfrascado en iniciativas similares y han fortalecido y creado sus propias unidades de verificación de datos. En esta línea, destacan: El Confidencial cuenta con el blog La Chistera, que comenzó como un experimento para seguir las sesiones de control del Congreso de los Diputados, pero que ha acabado ampliándose a las afirmaciones de políticos en otros foros. En 2016, Periodista digital pone en marcha la plataforma Verdad o Mentira y, desde 2017, el programa de televisión El Objetivo de La Sexta evalúa las declaraciones de políticos, una iniciativa que les ha servido para formar parte del International Fact Checking Network IFCN por la calidad del formato. Ese mismo año, eldiario.es lanza El Cazabulos, y con una actividad menor, pero con mayor profundidad en el análisis, El País cuenta desde 2017 con Hechos, un blog coordinado por Patricia R. Blanco que también investiga y desmiente algunas de las falsedades más relevantes. La web de Verne, de El País, también ha creado El Tragabulos, una página de Facebook donde se desmienten las noticias falsas que circulan por WhatsApp, Facebook y Telegram.

Estas unidades han servido de estímulo para que se creen en la red otros proyectos periodísticos independientes dedicados exclusivamente a la verificación de datos. De este modo, en 2006, nace de la mano de Alfredo Expósito la web Miniver. Ministerio de la Verdad, que en 2017 recibe el respaldo de Google al ser el primer portal español en mostrar la etiqueta de verificación de datos en los resultados de búsqueda. Siete años más tarde, en 2013, los periodistas Clara Jiménez y Julio Montes crean Maldita Hemeroteca, un proyecto independiente que se dedica sistemáticamente al desmentido y la verificación de noticias y que colabora con La Sexta y eldiario.es. De esta iniciativa matriz, surge en 2015 la web Maldito Bulo, «cuyo fin es dotar a los lectores de herramientas para que no te la cuelen», según se definen en su página web. Ambas propuestas se agrupan bajo en nombre de Maldita. En paralelo, destaca desde 2018 la startup de contenido audiovisual Newtral Media Audiovisual, fundada por la periodista Ana Pastor. 
Al hilo de estas propuestas, Graves y Cherubini (2016) optan por diferenciar entre dos modelos. Uno con una adscripción de tipo más profesional, asociada a los medios tradicionales, y otro de carácter alternativo o sin ánimo de lucro. En el caso de los proyectos independientes se han creado en el seno de lo que se denomina "periodismo emprendedor» (Manfredi Sánchez; Rojas Torrijos; Herranz de la Casa, 2015), y están alejados del modelo industrial cuyo accionariado corresponde a grandes grupos de comunicación. Por el contrario, las unidades activas de verificación de datos dependientes de los medios de comunicación están respaldas por los propios recursos financieros y humanos de los medios, por lo que cuentan con la ventaja de disponer de plataformas y recursos para su desarrollo, aunque también de las limitaciones de estar adscritos a una línea editorial.

\section{Metodología}

En este complejo marco informativo, las preguntas acerca de las políticas y estrategias contra las noticias falsas son: Q1: ¿Existen políticas y estrategias de servicio público para luchar contra la desinformación?; Q2: ¿Qué tipos de medidas han emprendido las radiotelevisiones públicas para ganar la batalla a las noticias falsas?; Q3: ¿Deben liderar los medios audiovisuales de servicio público la lucha contra la desinformación?

El objetivo de esta investigación es analizar las iniciativas y estrategias que las radiotelevisiones públicas europeas están aplicando para potenciar la lucha contra las noticias falsas.

El diseño metodológico para alcanzar este propósito parte de la revisión sistemática de la literatura científica, que forma parte de la investigación secundaria (Codina, 2017), y que ha permitido conocer las principales aportaciones al estado de la cuestión y generar un relato sobre este tema, que es de interés creciente en el área de Comunicación, pero aún no exhaustivamente abordado. De igual modo, se analiza la literatura gris (memorias, informes y otros documentos), así como las acciones desarrolladas por las televisiones públicas que son objeto de estudio. Se han analizado las memorias, informes y webs de las televisiones públicas de la Unión Europea, si bien, solo se enumeran aquellas prácticas e iniciativas más sobresalientes que 


\section{Las nuevas narrativas, en el entorno social \\ Universidad de La Laguna, diciembre de 2019}

destacan bien, por su originalidad y novedad, bien por su reconocimiento en la Academia.

Las políticas y estrategias contra las noticias falsas emprendidas por los medios audiovisuales de servicio público constituyen una parte importante en los medios de comunicación. Es por ello que cada vez son más los autores que establecen la necesidad de desterrar de la academia la perspectiva del centralismo de las redacciones para centrarse en manifestaciones del sector que van más allá de las formas del periodismo tradicional (Deuze; Witschge, 2017).

\section{Resultados}

Las televisiones públicas deben jugar un papel fundamental en la lucha contra las noticias falsas. La Unión Europea de Radiodifusión avaló en 2012 la posición estratégica que tenían que adoptar los medios audiovisuales de servicio público en la alfabetización mediática, demostrando su importante valía en la sociedad (Radoslavov, 2013). La misma posición defiende la profesora Bárbara Thomass (2004), que, entre otras, señala como funciones propias del servicio público audiovisual, la provisión de conocimiento e información, la superación de la brecha digital, el acceso a la información y el conocimiento, la promoción de la alfabetización mediática y la regulación de la sociedad de la información.

Las televisiones públicas europeas de forma reciente han desarrollado estrategias variadas para combatir las noticias falsas. Prácticamente, todas ellas, ofrecen en sus webs noticias o incluso secciones sobre noticias falsas. No obstante, algunas de ellas están apostando por iniciativas más complejas como decálogos o guías, sistemas de verificación, proyectos educativos para informar a los escolares y estudiantes sobre información confiable, videojuegos o, incluso, documentales falsos.

- Decálogos. Algunas de las radiotelevisiones públicas europeas optan por publicar decálogos o guías para que los usuarios interesados puedan aprender a localizar fake news o noticias falsas. Lo hace ZDF (Alemania) que introduce una web para enseñar, en especial al público más joven, la identificación de mentiras. Algunas de las televisiones pertenecientes a ARD 


\begin{abstract}
(Alemania), como BR y SWR, ofrecen tutoriales para identificar la desinformación en línea. Es la misma medida que han adoptado las televisiones públicas autonómicas en España que, a través de la Federación de Organismos de Radio y Televisión Autonómicos (FORTA), han lanzado un decálogo para detectar noticias falsas.
\end{abstract}

- Conferencias y debates. Una de las iniciativas por las que han apostado algunas de las televisiones públicas europeas para luchar contra la desinformación en línea, es organizar debates, seminarios o coloquios. Destaca en este sentido, el proyecto de la BBC (Reino Unido) Beyond Fake News (2018), a través del cual se ofrecieron conferencias en India y Kenia con el objeto de discutir desafíos y posibles soluciones a la desinformación en línea. En ese mismo año, RTÉ en Irlanda, invitó al público de la cadena a un debate para explorar el papel y el impacto de las noticias falsas en la sociedad. En 2019, CIRCOM, asociación de televisiones públicas regionales europeas, organizó en Roma el taller "Fake News y contraste de noticias" al que asistieron periodistas de medios audiovisuales de Bulgaria, Alemania, Italia, República Checa, Francia, Malta Serbia, Cataluña y Galicia (CRTVG, 2019).

- Sistemas de verificación. Algunas de las televisiones públicas ofrecen mecanismos de verificación de noticias falsas. La mayoría optan por una sección donde se comprueban noticias falsas extendidas en las redes sociales. En el caso vasco (EITB, en España), además se incorpora una sección semanal dirigida por el Responsable de Big Data en la Universidad de Deusto, para informar sobre posibles fake news. BBC, en Reino Unido, ha creado un equipo de verificación permanente, Reality Check, dedicado a examinar hechos y afirmaciones que se encuentran detrás de una historia o noticia con el objeto de determinar si son verdad o mentira. Se admiten denuncias y preguntas de los espectadores sobre noticias que encuentren en internet y en las redes sociales.

En Bélgica, RTBF, ofrece a sus ciudadanos la herramienta Faky para luchar contra la desinformación. Su novedad radica en que ésta se encuentra disponible en la web faky.be, pero se ofrece también una aplicación para instalar directamente en los smartphones. Para verificar una información, basta con copiar la URL del artículo en el programa y este ofrece el nivel de 


\section{Las nuevas narrativas, en el entorno social \\ Universidad de La Laguna, diciembre de 2019}

confiabilidad del mismo en una escala del 1 al 5 . En el caso de imágenes, la herramienta enumera los sitios donde se ha publicado una fotografía idéntica y los clasifica en base a la relevancia de la fuente (RTBF, 2019).

- Proyectos y programas educativos. Los menores son uno de los sectores de la sociedad que más preocupa a los responsables de las televisiones públicas para acabar con las noticias falsas. VRT (Bélgica) ha creado, junto con varios centros especializados (Mediawijs, IMEC, Voetweg 66 y Arteveldehogeschool) la herramienta EDUbox para informar a los jóvenes sobre política y democracia y ayudarlos a conocer la información manipulada. En Alemania, algunas de las entidades que componen ARD, y en Dinamarca, DR, publican información en sus webs para que pueda ser utilizada por los profesores en sus clases. En el caso de SR, también en Alemania, varios periodistas de la cadena pública visitan las escuelas para dar consejos a los estudiantes.

Otras televisiones, como France Télévisions (Francia), apuestan por programas infantiles especializados en alfabetización mediática, en donde se da respuesta a las preguntas de los menores, se les enseña a navegar por la web 2.0 o se les tutoriza para conocer profesiones como el periodismo o la informática. RTP en Portugal ha lanzado un telediario para niños de entre 8 y 12 años para incentivar el pensamiento crítico de los menores. En España, CMM (Castilla-La Mancha) ha incorporado el término fake news en su diccionario digital.

BBC (Reino Unido) apuesta por la participación creativa de los más jóvenes a través de Young Reporter, con la finalidad de empoderarlos a través del relato de sus propias historias. El objetivo final es trabajar, en colaboración con escuelas y organizaciones juveniles para proporcionar a los jóvenes las habilidades necesarias para comprender el funcionamiento de los medios de comunicación (BBC, 2019).

- Videojuegos. Con el objeto de acercar el conocimiento de las fake news a la sociedad, algunas televisiones públicas han desarrollado videojuegos y juegos interactivos. El equipo de Yle's News Lab (Finlandia) ha creado Troll Factory, un juego para que los usuarios conozcan qué papel desempeñan las redes sociales en la difusión de noticias falsas. Para ello, se combina contenido 


\section{Las nuevas narrativas, en el entorno social}

Universidad de La Laguna, diciembre de 2019

auténtico de redes sociales con una simulación personalizada en función de las elecciones del usuario (Yle, 2019).

SWR (Alemania) apuesta por Fake Finder. El usuario recibe varios mensajes para que descubra si son noticias verdaderas o falsas. Para ello, cuenta, en ocasiones con un asistente virtual, que guía a los jugadores y les brinda consejos y recomendaciones.

- Perfiles falsos y falsos documentales. Dentro de las iniciativas más arriesgadas, encontramos la emisión de falsos documentales, como "Desmontando a Goya”, en CARTV (Aragón), reconocido por el Consejo de la Academia de las Ciencias y las Artes de Televisión. En el documental se vinculan las obras de Goya con Cataluña con el propósito de hacer reflexionar al espectador (CARTV, 2018). EITB, en el País Vasco, puso en marcha un experimento para demostrar la facilidad con la que se viralizan las fake news en Internet a través de un perfil falso en Twitter, en el programa 360 grados. El objetivo en ambos casos, era hacer reflexionar al público acerca de la rapidez en la que se transmiten falsos contenidos en la sociedad actual.

\section{Discusión y conclusiones}

La investigación desarrollada permite dar por conseguido el objetivo planteado inicialmente de analizar las iniciativas y estrategias que las radiotelevisiones públicas europeas están aplicando para potenciar la lucha contra las noticias falsas. Los medios audiovisuales de servicio público referenciados en el marco de este trabajo han sido ZDF, SR, SWR y ARD, en Alemania; BBC en Reino Unido; RTÉ en Irlanda; RTBF y VRT en Bélgica; DR en Dinamarca; y France Télévisions en Francia. En el caso de España se han reseñado tres televisiones públicas: EITB (País Vasco); CMM (Castilla-La Mancha); CARTV (Aragón).

Estos medios cuentan con secciones especializadas sobre noticias falsas. También apuestan por otras iniciativas más complejas para mostrar la realidad de las fake news y luchar contra ellas. En este sentido, destacan los decálogos, las conferencias y debates, los sistemas de verificación, los proyectos y programas educativos, así como los videojuegos y los falsos documentales. Todas estas iniciativas ayudan a desmentir las noticias falsas y a fomentar una 


\section{Las nuevas narrativas, en el entorno social}

\section{Universidad de La Laguna, diciembre de 2019}

cultura de transparencia y responsabilidad, aclarando dónde se encuentran las inexactitudes y aportando datos originales que construyan un relato verídico.

Las políticas y estrategias analizadas surgen a partir de 2012, después de que la Unión Europea de Radiodifusión avalase la posición estratégica que deben adoptar los medios audiovisuales de servicio público en la alfabetización mediática, demostrando su importante valía en la sociedad (Radoslavov, 2013). Desde entonces las radiotelevisiones públicas europeas no han parado de personalizar sus servicios para responder a las demandas de medios de comunicación y usuarios, que apuestan por textos periodísticos contrastados a partir de fuentes fiables, documentos oficiales y resultados de investigaciones solventes.

Esta situación refleja el cambio cultural y tecnológico que está atravesando la industria mediática (Pavlik, 2015) y evidencia la voluntad de consolidar estas propuestas de servicio público, que priorizan la agenda cívica y la vigilancia de los poderes públicos, cuando el auge de las llamadas fake news y la posverdad amenaza los estándares de calidad y la credibilidad de medios y periodistas (Lewandowsky; Ecker; Cook, 2017).

Los medios son conscientes de que deben garantizar la credibilidad de la información que transmiten, así como alertar de la desinformación. Al hilo de estos planteamientos, quedan respaldadas las teorías de Amorós (2018) y Mantzarlis (2018), que defienden que estas estrategias son la mejor forma de contrarrestar el poder de las noticias falsas y han sido ideadas por los medios para combatirlas y ofrecer a la ciudadanía un relato completamente ajustado a la realidad. Por este motivo, los servicios audiovisuales públicos deben seguir apostando por la verificación de datos, en un momento en el que el entorno digital está definido por la desinformación y la proliferación de informaciones falsas.

\section{Referencias bibliográficas}

Adair, B. \& Thakore, I. (2015). Fact-checking Census Finds Continued Growth Around the World. Duke Reporters'Lab. Disponible en: http://cort.as/$\underline{\mathrm{RVzC}}$. 
Las nuevas narrativas, en el entorno social

Universidad de La Laguna, diciembre de 2019

Allcott, H. \& Gentzkow, M. (2017). Social Media and Fake News in the 2016

Election. Journal of Economic Perspectives, v. 31, n. 2, pp. 211-236

(https://www.doi.org/10.1257/jep.31.2.211).

Amorós, M. (2018). Fake News. La verdad de las noticias falsas. Barcelona: Plataforma Editorial.

Ayuso, B. \& Bauzá, B. (2018). Almanaques, espías y un chándal: el universo secreto de los fact-checkers. Jot Down, n. 31 (abril), pp. 4-13.

Balmas, M. (2012). When Fake News Becomes Real. Combined Exposure to Multiple News Sources and Political Attitudes of Inefficacy, Alienation, and Cynicism. Communication Research, v. 41, pp. 430-454

(https://doi.org/10.1177/0093650212453600).

BBC (2019). What is BBC Young Reporter? Disponible en: https://cutt.ly/mra7gl9

Catalina-García, B., Sousa, J. P. \& Silva, L. C. (2019). Consumo de noticias y percepción de fake newsentre estudiantes de Comunicación de Brasil, España y Portugal. Revista de Comunicación, v.18, n. 2, pp. 93-115 (https://doi.org/10.26441/RC18.2-2019-A5).

CARTV (2018). La Academia de la TV concede un accésit al documental de Aragón TV "Desmontando a Goya”. Disponible en: https://cutt.ly/Urq7hNn Champy, F. (2011). Nouvelle Théorie Sociologique des Professions. Paris: Presses Universitaires de France.

Cherubini, F. \& Graves, L. (2016). The rise of fact-checking sites in Europe. Reuters Institute for the Study of Journalism, University of Oxford. Codeluppi, V (2018). II tramonto della realtà. Come i media trasformano le nostre vite. Milano: Carocci.

Codina, L. (2017). Revisiones sistematizadas y cómo llevarlas a cabo con garantías: Systematic reviews y SALSA Framework. Disponible en: https://cutt.ly/7wXGp1b

CRTVG (2019). Xornalistas de televisión europeas debateron en Roma sobre o problema da credibilidade e das "fake news". Disponible en: https://cutt.ly/7rq7j2y

Derakhshan, H. \& Wardle, C. (2017). Information Disorder: Definitions. In AA.VV., Understanding and Addressing the Disinformation Ecosystem (pp. 


\section{5-12). Pennsylvania: Annenberg School for Communication.}

Deuze, M., \& Witschge, T. (2017). Beyond journalism: Theorizing the transformation of journalism. Journalism, v.19, n.2, pp. 165-181 (https://doi.org/10.1177/1464884916688550).

Duke Reporter's Lab. (2019). Fact-Checking. Disponible en: http://cort.as/$\underline{\mathrm{ROgV}}$

Fisher, C. (2018). What is meant by 'trust' in news media? In K. Otto \& A. Köhler (Eds.), Trust in Media and Journalism (pp. 19-38). Berlin: Springer. Graves, L. (2016). Deciding What's True. New York: Columbia University Press. Graves, L. \& Cherubini, F. (2016). The rise of fact-checking sites in Europe. Reuters Institute Digital News Report. Disponible en: https://cutt.ly/heVfOH3

Greenberg, D. (2017). Deciding What's True: The Rise of Political FactChecking in American Journalism. Journal of Communication, v. 67 (https://doi.org/10.1111/jcom.12329).

Gueham, F. (2017). Le fact-checking: une réponse à la crise de l'information et de la démocratie. Paris: Fondation pour l'innovation politique.

Haigh, M.; Haigh, T. \& Kozak, N.-I. (2017). Stopping Fake News. The work practices of peer-to-peer counter propaganda. Journalism Studies, 25 de abril, pp. 1-26 (https://doi.org/10.1080/1461670X.2017.1316681).

Jankowski, N.W. (2018). Researching Fake News: A Selective Examination of Empirical Studies. Journal of the European Institute for Communication and Culture, v. 25, pp. 248-255 (https://doi.org/10.1080/13183222.2018.1418964).

Khaldarova, I. \& Pantti, M. (2016). Fake News. The narrative battle over the Ukrainian conflicto. Journalism Practice, 12 de abril, v. 10, pp. 891-901 (https://doi.org/10.1080/17512786.2016.1163237).

Lewandowsky, S., Ecker, U. K. \& Cook, J. (2017). Beyond Misinformation: Understanding and Coping with the "Post-Truth" Era. Journal of Applied Research in Memory and Cognition. Disponible en: http://cort.as/-RaYR López-Vidales, N.; González Aldea, P. \& Medina de la Viña, E. (2011). Jóvenes y televisión en 2010: Un cambio de hábitos. Zer, Revista de Estudios de Comunicación, v. 16, n. 30, pp. 97-113. 
Las nuevas narrativas, en el entorno social

Universidad de La Laguna, diciembre de 2019

Manfredi Sánchez, J. I.; Rojas Torrijos, J. L. \& Herranz de la Casa, J. M. (2015). Innovación en el periodismo emprendedor deportivo. Modelo de negocio y narrativas. El profesional de la información, v. 24, n. 3, pp. 265-273.

Mantzarlis, A. (2018). Fact-checking 101. In: Ireton, Cherilyn; Posetti, Julie. Journalism, fake news \& disinformation: Handbook for journalism education and training. Francia: Unesco, pp. 85-100.

McChesney, R. (2014). Blowing the Roof off the Twenty-First Century. New York: Monthly Review Press.

Mihailidis, P. \& Viotty, S. (2017). Spreadable spectacle in digital culture: Civic expression, fake news, and the role of media literacies in 'post-fact' society. American Behavioral Scientist, 61(4), pp.441-454 (https://doi.org/10.1177/0002764217701217).

Palau Sampio, D. (2018). Fact checking y vigilancia del poder: La verificación del discurso público en los nuevos medios de América Latina. Communication \& Society, v. 31, n. 3, pp. 347-365 (https://doi.org/10.15581/003.31.3.347-365).

Pavlik, J. V. (2015). Transformation: examining the implications of emerging technology for journalism, media and society. Athens $J$ Mass Media Commun, v.1, n. 1, pp. 9-24.

Petroni, S., Massa, A., \& Anzera, G. (2017). Lo specchio di Aletheia: Fake news e politica internazionale (V. 1). Edizioni Nuova Cultura.

Radoslavov, S. (2013). Media Literacy Promotion as a Form of Public Value? Comparing the Media Literacy Promotion Strategies of the BBC, ZDF and RAI. En Ferrell Lowe, G. y Martin F. (eds.). The Value of Public Service Media (pp. 205-222). Göteborg: Nordicom.

Rapp, D. N. \& Salovich, N. A. (2018). Can't We Just Disregard Fake News? The Consequences of Exposure to Inaccurate Information. Policy Insights from the Behavioral and Brain Sciences, v. 5, n. 2, pp. 232-239 (https://doi.org/10.1177/2372732218785193).

RTBF (2019). Faky, nouvel outil RTBF pour lutter contre la désinformation, suspendu, reviendra dans une version améliorée. Disponible en: https://cutt.ly/Krq7bWX 
Rubin, V. L., Chen, Y. \& Conroy, N. J. (2016). Deception Detection for News:

Three types of Fakes. ASSIST, v. 52, n. 1, pp. 1-4.

Schudson, M. \& Zelizer, B. (2017). Fake News in context. In AA.VV.,

Understanding and Addressing the Disinformation Ecosystem (pp. 1-4).

Pennsylvania: Annenberg School for Communication.

Spivak, C. (2011). The fact-checking explosión. American Journalism Review v.

32, n. 4, pp. 38-43.

Stencel, M. (2016). Global Fact-Checking up 50\% in Past Year. Duke

Reporters's Lab. Disponible en: http://cort.as/-RRUO

Tandoc, E. C.; Lim, Z. W. \& Ling, R. (2018). Defining 'Fake News': a typology of

scholarly definitions. Digital Journalism, v. 6, n. 2, pp. 137-153

(https://doi.org/10.1080/21670811.2017.1360143).

Thomass, B. (2004). The Agenda of the world summit on the information society and PSB. RIPE Conference 2004. Disponible en: https://cutt.ly/qrq7nok

Vázquez Herrero, J.; Vizoso, A. \& López García, X. (2019). Innovación

tecnológica y comunicativa para combatir la desinformación: 135

experiencias para un cambio de rumbo. El profesional de la información,

v. 28, n. 3 (https://doi.org/10.3145/epi.2019.may.01).

Watts, D. J. \& Rothschild, D. M. (2017). The minority report on the fake news

crisis: (spoiler alert: it's the real news). In AA.VV., Understanding and

Addressing the Disinformation Ecosystem (pp. 23-37). Pennsylvania:

Annenberg School for Communication.

Yle (2019). Yle's "troll factory" game unmasks how fake news spreads via social media. Disponible en: https://cutt.ly/nrq7m6a

Zaryan, S. (2017). Truth and Trust: How audiences are making sense of Fake News. Lund: Lund University.

Zommer, L. (2014). El boom del fact checking en América Latina. Aprendizajes

y desafíos del caso de Chequeado. Konrad Adenauer Stiftung.

* Este artículo forma parte de las actividades del proyecto de investigación (RTI2018-096065-B-I00) del Programa Estatal de I+D+l orientado a los Retos de la Sociedad del Ministerio de Ciencia, Innovación y Universidades y del Fondo Europeo de Desarrollo Regional (FEDER) sobre "Nuevos valores, gobernanza, financiación y servicios audiovisuales públicos para la sociedad de Internet: contrastes europeos y españoles". 